England. The results showed that complex partial seizures, with or without secondary generalization, responded best with more than $1 / 2$ achieving a greater than $50 \%$ reduction. Generalized tonic-clonic seizures also improved. Absence or myoclonic seizures were not benefited. Only 4 patients $(10 \%)$ were seizure free on monotherapy with vigabatrin (Gibbs JM et al. Vigabatrin in intractable childhood epilepsy: a retrospective study. Pediatr Neurol Sept/Oct 1992; $\underline{8}: 338$ 40).

In a study of 45 patients ages $2-21$ years treated with vigabatrin in Paris, France, 20 (44\%) showed no benefit and $24 \%$ were well controlled. Partial seizures were most responsive.

\title{
ANTICONVULSANTS AND SERUM CHOLESTEROL
}

The effect of antiepileptic drugs (AED) on total cholesterol, high density lipoprotein (HDL) cholesterol and triglycerides was studied in 208 epileptic children compared with 175 normal children at the University of Bologna, Italy. In epileptic patients the mean total cholesterol plasma level was higher $(195 \mathrm{mg} / \mathrm{dl})$ than that of controls $(184 \mathrm{mg} / \mathrm{dl})$. HDL cholesterol and triglyceride levels were not different in the two groups. Carbamazepine and phenobarbital treated patients had significant elevations of mean cholesterol values and valproate treated patients had a significant lowering of cholesterol. An increase in cholesterol in the primidone and phenytoin treated patients was not significant. In a group of 32 patients studied before and during AED therapy the total cholesterol level before therapy was $168 \mathrm{mg} / \mathrm{dl}$ and after therapy $203 \mathrm{mg} / \mathrm{dl}$. The highest level $(313 \mathrm{mg} / \mathrm{dl})$ of total cholesterol was observed in a boy receiving carbamazepine therapy (Franzoni E et al. Total cholesterol, high-density lipoprotein cholesterol, and triglycerides in children receiving antiepileptic drugs. Epilepsia Sept/Oct 1992; 33:932-935). (Reprints: Professor E. Franzoni, Centro di Neurologia Pediatrica, Universita di Bologna, Via Massarenti 11, 4013B Bologna, Italy.)

COMMENT. Carbamazepine treated patients with a family history of hypercholesterolemia or cardiac infarction should be followed carefully for elevated levels of total cholesterol. The effect of antiepileptic drugs on cholesterol levels during treatment with the ketogenic diet should be carefully monitored, especially when treatment is combined with carbamazepine or phenobarbital.

Carbamazepine appeared to be responsible for a nonconvulsive status epilepticus in a 12 year old epileptic girl, in a report from Washington University School of Medicine, St. Louis, MO (Callahan DJ, Noetzel MJ. Neurology Nov 1992; $42: 2198)$. CBZ level was 7.1 $\mathrm{mcg} / \mathrm{ml}$ on admission. The absence status was resistant to multiple AEDs and was associated with increased intracranial pressure and transiet MRI abnormalities. CBZ should be avoided in patients with generalized, synchronous, spike-and-wave EEG patterns. 